\title{
Anomalous Pulsation of Field RR Lyrae Variables: Photometric and Spectroscopic Study of CM Leo, BS Com, and CU Com
}

\author{
A. Bragaglia, G. Clementini, L. Di Fabrizio, S. Di Tomaso, R. Merighi, \\ M. Tosi \\ Osservatorio Astronomico di Bologna, via Ranzani 1, I-40127 Bologna, \\ Italy
}

I. Evans, C. Sneden, R. Wilhelm

Department of Astronomy and McDonald Observatory, The University of Texas at Austin, TX, USA

H. Smith

Michigan State University, Dept. of Physics \& Astronomy, East Lansing, MI, USA

\begin{abstract}
We present results on three presumed "anomalous" RRab stars: one is found to be an RRc variable, and the two others need further study.
\end{abstract}

\section{Are CM Leo, CU Com, and BS Com Truly Anomalous?}

The three field RR Lyrae variables CM Leo, CU Com, and BS Com had been defined as "anomalous" by Castellani, Maceroni, \& Tosi (1983), since they deviated from the general period-height (on the Galactic plane) relation, hence from the metallicity-height relation. Clementini et al. (1995) presented a first study of these (and five more) stars, aimed at finding whether their anomaly was real, or simply due to mis-classifications in type, period, or amplitude. No immediate explanation was found for these three, since their light curves were complicated and badly sampled. Possible explanations for the anomalies observed in the light curves are: (i) double-mode pulsation, (ii) Blazhko effect, or (iii) binarism.

We have conducted further observations, but the only star for which we have concluded the observational campaign is CM Leo. We have built BVI light curves (using the Loiano 1.5-m telescope, with a contribution from the Michigan State University $60-\mathrm{cm}$ telescope for CM Leo), and derived high accuracy radial velocity curves (from $R=30,000$ spectra taken at the $2.7-\mathrm{m}$ telescope of the McDonald Observatory). We use differential photometry between our targets and some comparison stars found in the same field of view to define the light curves, which are then calibrated to the standard magnitude system on a photometric night. The radial velocities have been derived using the cross-correlation technique. 
Our ultimate goal is to derive information on the stellar parameters (mass, mass-metallicity relation), structure (magnetic fields, etc.), pulsation mechanism (resonance, mode mixing), and evolution of these horizontal branch stars. As a bonus, precise metal abundances for the program stars will also be derived from the spectra taken at minimum light.

\subsection{Leo}

We have obtained $456 \mathrm{~V}$ data points (and about 100 in $B$ and $I$ ), from which period and epoch were redetermined $(P=0.361699 \mathrm{~d} ; E=J D 2450841.3633853)$. The presumed anomaly was solved, since the star is type RRc, rather than RRab; there is a possibility that its pulsation mode has changed after classification in the GCVS. From a residual scatter in the light curve, a secondary periodicity of Blazhko type with small amplitude and large period could be present.

We also obtained 12 high-resolution spectra on two consecutive nights, covering all the cycle. The radial velocity curve has the typical shape and amplitude $\left(26.5 \mathrm{~km} \mathrm{~s}^{-1}\right)$ expected for an RRc star. Results on this star will be presented in Di Fabrizio et al. (in preparation).

\subsection{CU Com}

For CU Com we only have about $300 \mathrm{~V}$ measures up to 1999 , and about one fifth of that in $B$ and $I$. The $V$ light curve amplitude $(0.65 \mathrm{mag})$ is appropriate for an RRc star, but a lot of scatter is present, and the curve is reminiscent of a double mode pulsator; the Blazhko explanation is less probable since the light curve changes night by night. More data are required.

We obtained 15 high-resolution spectra, but we could not cover the entire cycle; the radial velocity curve amplitude is at least $32 \mathrm{~km} \mathrm{~s}^{-1}$, but it is not evident that this is a typical RRc type rather than an RRab type curve.

\subsection{BS Com}

For BS Com we have about $240 \mathrm{~V}$ data points, and some tens of $B$ and $I$ points. The light curve appearance and amplitude $(\simeq 0.7 \mathrm{mag})$ would classify it as RRab type, but there is a lot of scatter, and what looks like two different curves near maximum light. The star has been classified either RRc or RRab, and has been noticed to vary in its light curve, possibly due to double mode pulsation, since BS Com changes its light curve on consecutive nights.

The radial velocity curve, derived from 14 spectra taken on two contiguous nights, is not decisive in assigning a type to this RR Lyr star: it does not cover all the cycle, and could also be thought to be composed of two different curves for the two nights.

\section{References}

Castellani, V., Maceroni, C., \& Tosi, M. 1983, A\&A, 128, 64

Clementini, G., Tosi, M., Bragaglia, A., Merighi, R., \& Maceroni, C. 1995, MNRAS, 275, 929 